\title{
GEOQUÍMICA DE ISÓTOPOS DE CARBONO E OXIGÊNIO DOS CARBONATITOS DO COMPLEXO ALCALINO DE MATO PRETO, PARANÁ, BRASIL
}

\author{
ROBERTO VENTURA SANTOS*, MARCEL AUGUSTE DARDENNE** e \\ EIICHIMATSUI***
}

\begin{abstract}
CARBON AND OXYGEN STABLE ISOTOPE GEOCHEMISTRY OF THE CARBONATITES FROM THE MATO PRETO ALKALINE COMPLEX, PARANA, BRAZIL. It is presented and discussed the $\mathrm{C}$ and $\mathrm{O}$ stable isotopes analyses of the carbonatites of the Mato Preto Alkaline Complex, Paraná, Brazil. There are two carbonatites generation in the complex. The main one is dominantiy calcitic and presents $\mathrm{C}$ and $\mathrm{O}$ isotopic values ranging besteen $-3 \% \mathrm{o}-0.0 \% \mathrm{o}$ and $+11.0 \% \mathrm{o}-+16.0 \% \mathrm{o}$ respectively. The samples show a linear trend when plotted in a $\delta \mathrm{O}^{18}$ SMOW versus $8 \mathrm{C}$ PDB diagram, which may represent a Rayleigh distillation process. The older carbonatitic generation is mainly dolomitic and shows lower $\mathrm{C}$ and 0 isotopes values than the calcitic one. Some samples that were analyzed along the borehole DB47 show inexpressive variations of the isotopic values. The spatial distribution of the isotope data over the complex suggests that the carbonatites were isotopicaly contaminated by the host rocks.
\end{abstract}

Keywords: Stable isotopes, carbonatites, Mato Preto Alcaline Complex.

RESUMO São apresentados os dados de isótopos estáveis de $\mathrm{C}$ e $\mathrm{O}$ em carbonates dos carbonatitos do Complexo de Mato Preto, Paraná. Dentre as gerações de carbonatito, a principal possui composição calcítica e apresenta valores de isótopos estáveis de C e $\mathrm{O}$ que variam entre $-3,0 \% \mathrm{o}-0,0 \% \mathrm{o}$ e $+11,0 \% \mathrm{o}-$ $+16,0 \% \mathrm{o}$, respectivamente, considerando-se as amostras de carbonatito fresco. Ao se observar estas amostras no diagrama $\delta \mathrm{O}^{18}$ smow versus $\delta \mathrm{C}^{13}$ PDB, verifica-se que se dispõem segundo uma tendência linear, obedecendo à destilacão de Rayleigh. A geração mais precoce é dominantemente dolomítica e apresenta valores de $\delta \mathrm{O}^{18}$ e $\delta \mathrm{C}^{13}$ mais baixos que a geração de carbonatito calcítico tardio. Ao se analisar o furo de sondagem DB47, observam-se variações inexpressivas dos dados isotópicos com a profundidade de coleta das amostras. A distribuição espacial das amostras sugere, entretanto, a existência de contaminação por parte dos granites e metassedimentos encaixantes.

Palavras-chaves: Isótopos estáveis, carbonatitos, Complexo Alcalino de Mato Preto.

INTRODUÇÃO O Complexo de Mato Preto situa-se a cerca de $100 \mathrm{~km}$ ao norte de Curitiba, no município de Cerro Azul (Fig. 1), e detém uma importante reserva de fluorita estimada em 2,16 milhões de toneladas de minério com teor médio de $65 \%$ de $\mathrm{CaF}_{2}$ (Jenkins 1987).

Este trabalho tem por objetivo apresentar dados de isótopos estáveis de $\mathrm{C}$ e $\mathrm{O}$ obtidos a partir dos carbonates dos carbonatitos do Complexo Alcalino Carbonatítico de Mato Preto, Paraná. Faz-se uma breve discussão teórica sobre a aplicação de isótopos em carbonatitos e em seguida procura-se interpretar os dados obtidos em função da geologia do complexo alcalino e dos processos de diferenciação em carbonatitos.

GEOLOGIA As duas principais unidades geológicas expostas na região de Cerro Azul são o Grupo Açungui e o Granito Três Córregos, ambos de idade pré-cambriana. As rochas fanerozóicas são representadas por intrusivas alcalinas, diques de diabásio, fonólitos e rochas afins. O Complexo de Mato Preto faz parte da Província Alcalina do Arco de Ponta Grossa (Almeida 1983) e aloja-se ao longo da Falha de Morro Agudo, no contato tectônico entre as duas unidades pré-cambrianas (Fig. 1).

A presença de rochas alcalinas na região de Cerro Azul é conhecida desde 1937 (Carvalho \& Pinto, In: Trein et al. 1966), enquanto os carbonatitos associados ao Complexo de Mato Preto foram pela primeira vez descritos por Loureiro \& Tavares (1983). Diques de fonólito que recortam o complexo foram datados pelo método $\mathrm{K}-\mathrm{Ar}$ e acusaram idades mínimas de 65,2 $\pm 3,3$ e 67,0 \pm 3,4 Ma (Cordani \& Hasui 1968).

$\mathrm{O}$ complexo alcalino estende-se por uma área aproximada de $12 \mathrm{~km}$ e possui quatro estruturas circulares principais, cada uma com cerca de $1 \mathrm{~km}$ de diâmetro (Unhas tracejadas na Fig. 2). As estruturas são circundadas por rochas graníticas e metassedimentares que se encontram afetadas por metassomatismo alcalino. Os dados de isótopos estáveis foram obtidos a partir de rochas situadas nas três estruturas circulares localizadas ao norte do Ribeirão Mato Preto, onde dominam sienito fenitizados e secundariamente carbonatitos calcíticos, fonólitos e rochas alcalinas ultramáficas. Os carbonatitos apresentam três formas de ocorrência principais: diques de carbonatito e brechas explosivas de conduto intrusivas e não intrusivas. Brechas de conduto não intrusivas foram definidas por Ulbrich (1986) como aquelas em que não se verifica o transporte ascendente dos clastos da rocha encaixante. As três formas de ocorrência provavelmente transicionam entre si e se relacionam a eventos contemporâneos. Na área do depósito de fluorita Clugger, foram identificadas duas gerações de carbonatitos associadas às brechas. Na primeira geração, o carbonato de composição dolomítica a anquerítica associa-se quase exclusivamente aos fragmentos das rochas feldspáticas, enquanto na segunda geração, o carbonato de composição calcítica ocorre na matriz de brechas ou preenchendo fraturas de fragmentos maiores (Santos 1988, Santos \& Dardenne 1988). Cabe ressaltar que esses carbonatitos foram observados principalmente na área do depósito de fluorita Clugger, restando dúvidas se a mesma estruturação é válida para as demais estruturas circulares. A falha de Morro Agudo separa as duas estruturas circulares, localizadas na porção noroeste da área e, segundo Fiori (1985), é o reflexo de falhas transcorrentes do embasamento cristalino. A falha, juntamente com outras estruturas regionais, teve um importante papel no alojamento do complexo e da própria mineralização de fluorita. A quarta

\footnotetext{
* Departamento de Geologia, Universidade Federal de Ouro Preto, CEP 35400, Ouro Preto, MG, Brasil e Department of Geophysical Sciences,

University of Chicago, 5734 S. Ellis, Chicago, II60637, EUA

** Instituto de Geociências, Universidade de Brasília, CEP 70910, Brasília, DF, Brasil

*** Centro de Energia Nuclear na Agricultura (CENA), Universidade de São Paulo, Caixa Postal 96, CEP 13400, Piracicaba, São Paulo, Brasil
} 
estrutura circular posiciona-se ao sul do Ribeirão Mato Preto e é constituída por fonólitos e brechas de conduto intrusivas (Santos 1988).

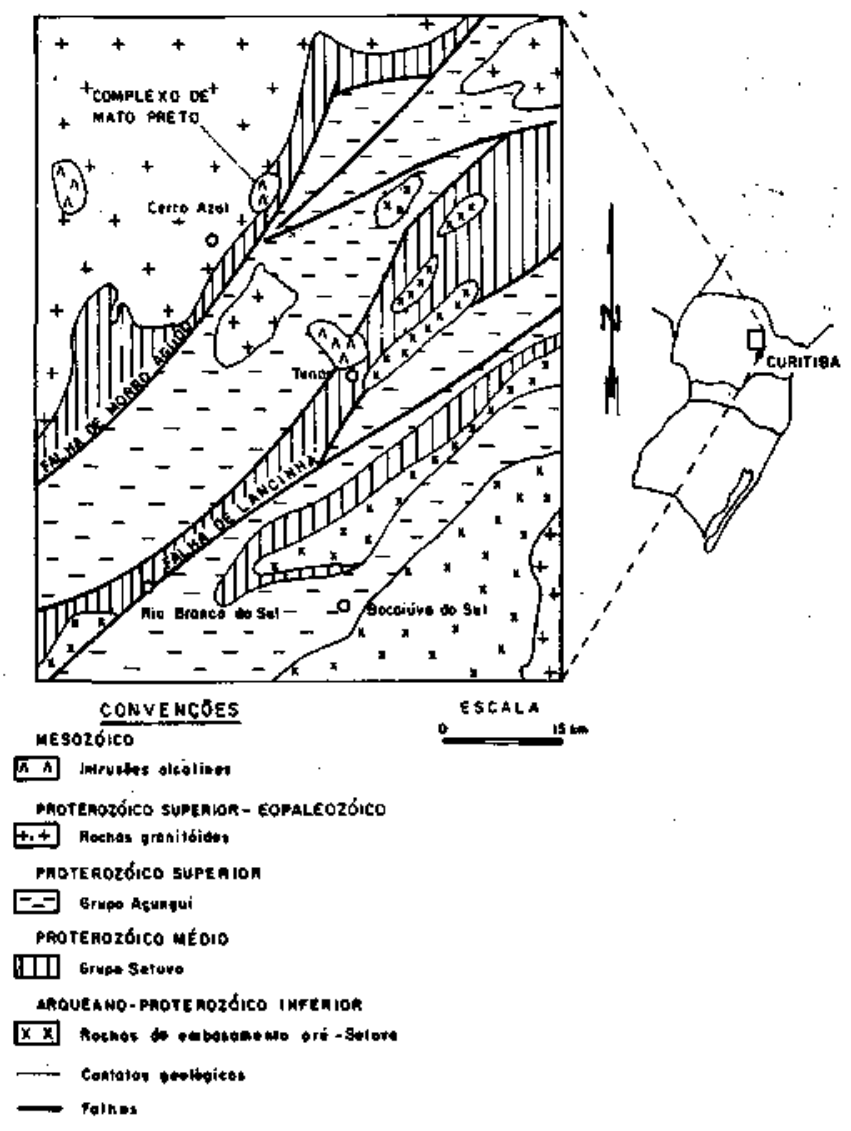

Figura 1-Mapa de lacalizaçāo

Figune 1 - Location map

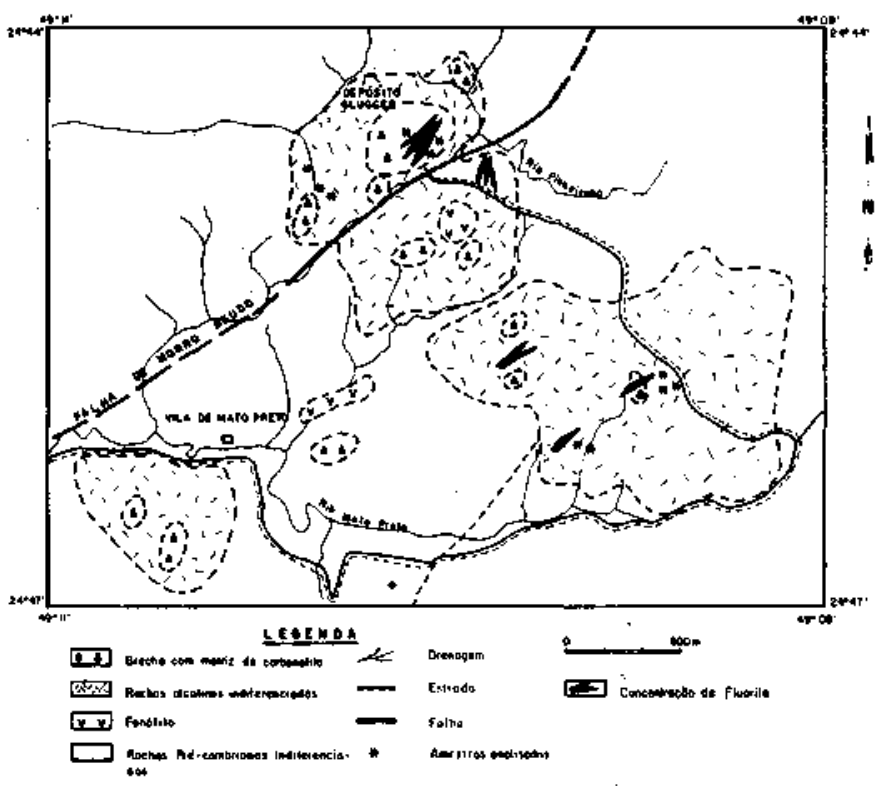

Figura 2 - Mapa geológico esquemático do Complexo de Mato Preto mostrando a estruturação geral do mesmo e o local de coleta das amostras de carbonatito para análise de isótopos estáveis

Figure 2 - Schematic geologic map of Mato Preto Complex showing its general structure and location of samples of carbonatite for stable isotopes analysis
GEOQUÍMICA DE ISÓTOPOS ESTÁVEIS A aplicação de isótopos estáveis em geologia baseia-se na diferença mínima de sua massa, que é função da quantidade de neutrons presentes em seu núcleo. A energia de uma molécula depende em parte da freqüência de vibração de seus átomos, que por sua vez é dependente de sua massa atômica. Assim, as moléculas de uma mesma substância que são constituídas por isótopos mais leves terão uma maior energia de vibração. Esta variação energética controla o fracionamento isotópico, ou seja, a partição isotópica entre as substâncias. Segundo Hoefs (1987), os principais fenômenos responsáveis pelo fracionamento isotópico são: a. reações de trocas isotópicas; b. processos cinéticos, que dependem principalmente da diferença na taxa de reação entre duas moléculas isotopicamente distintas; e c. processos físico-químicos.

Os dados de isótopos estáveis são apresentados mediante o parâmetro $\delta$ definido por:

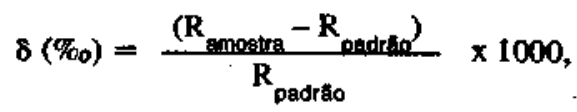

no qual, para o carbono e oxigênio, os valores de R correspondem respectivamente a $\mathrm{C}^{13} / \mathrm{C}^{12}$ e $\mathrm{O}^{18} / \mathrm{O}^{16}$. Para o carbono, utiliza-se normalmente o padrão PDB (Belemnitella americana da formação cretácea PeeDee, Carolina do Sul), enquanto que, para o oxigênio, utilizam-se os padrões PDB e SMOW. Neste trabalho será utilizado o padrão SMOW, que corresponde à composição isotópica média da água do mar.

A geoquímica de isótopos estáveis foi tema de trabalhos de Pillot (1974), White (1974), Nielsen \& Buchardt (1975) e Hoefs (1987), que descreveram seu comportamento frente aos processos geológicos.

A aplicação de isótopos estáveis ao estudo de carbonatitos foi feita por Taylor et al. (1967), Pineau \& Javoy (1969), Denayer (1970), Deines \& Gold (1973), Scheibe (1979), Torquato (1987), além de outros. Dentre esses, Taylor et al. (1967) foram dos primeiros a fazer um estudo sistemático sobre a composição isotópica dos carbonatitos, tendo definido que os carbonates dos carbonaíitos possuem valores de $\delta \mathrm{O}^{18}$ e $\delta C^{13}$ variando entre $+6 \%_{0}-+8,5 \%_{0}$ e $-8 \%_{0}-5 \%_{0}$ respectivamente.

Posteriormente, Deines \& Gold (1973) observaram que o retângulo definido por Taylor et al. (1967) era muito restrito para englobar a maior parte dos carbonatitos e concluíram que existem dois grupos de carbonatitos separáveis com base na distribuição dos valores de $\delta \mathrm{O}^{18}$ e $\delta \mathrm{C}$. Um grupo que corresponde aos carbonatitos subvulcânicos e que apresentam pequena dispersão dos valores isotópicos e um grupo representado pelo complexos carbonatfticos vulcânicos que mostram uma expressiva variação na composição isotópica. Para explicar esta característica, os autores levantaram as seguintes possibilidades:

a. Perda dos fluidos ricos em água isotopicamente mais leve na associação vulcânica durante a redução da pressão;

b. Equilíbrio da água magmática com os carbonatitos a baixa temperatura; e

c. Influência de águas meteóricas.

Em um trabalho anterior, Pineau \& Javoy (1969) já utilizavam a primeira e terceira hipóteses de Deines \& Gold (1973) para explicar variações dos valores isotópicos de $\mathrm{C}$ e $\mathrm{O}$ em carbonatitos. Assim, eles apresentam o diagrama da figura 3 no qual se encontra representado o retângulo de Taylor: uma reta ao longo da qual ocorre a evolução isotópica dos carbonatitos e uma zona cuja composição isotópica deve-se às alterações meteóricas. A reta mencionada corresponde à destilação de Rayleigh, mostrando que, em um sistema onde coexistam carbonato e $\mathrm{CO}_{2}$ em equilíbrio, o fracionamento isotópico pode ocorrer pela liberação preferencial do $\mathrm{CO}_{2}$ mais leve seguido por um novo reequilíbrio do sistema. Se esse 
processo agir interativamente, serão gerados carbonates cujos valores de $\mathrm{C}$ e $\mathrm{O}$ correspondem à reta da figura 3 .

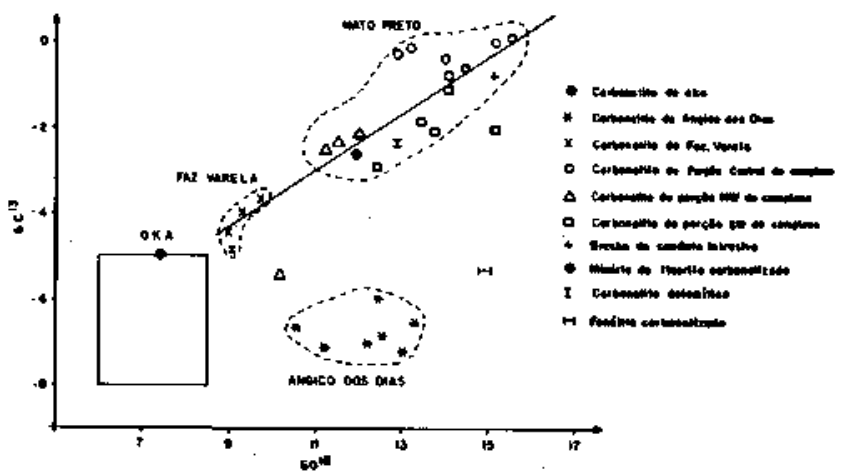

Figura 3 - Diagrama $8 O^{t 6}$ w. $8 C^{43}$ para os carbonatos dos carbonatilos dos complexos de Mato Preto, Angico dos Dias, Oka e Fazenda Varela. No diagrama encontra-se representado tambent o retangulo de Taylor (I968)

Figure $3-80^{78} \times 80^{13}$ diagram for carbonates of carbonatites of Maso Preto, Angico doe Dies, Oka and Varela Ferui complexos. This dingram also thows the Taylor (1968) sectamble

Composição isotópica dos carbonatitos de Mato Preto Foram feitas 29 análises de isótopos de carbono e oxigênio nos carbonates dos carbonatitos e dos mármores do Grupo Açungui (Tab. 1). As amostras foram coletadas em vários pontos do complexo e ao longo de um testemunho de sondagem, com a finalidade de verificar as variações isotópicas com a profundidade. Para identificar os carbonates e estabelecer a proporção aproximada de calcita e dolomita, utilizou-se a difratometria de raios $\mathrm{X}$ e uma solução acidulada à base de alizarina red S e ferricianeto de potássio (Hutchison 1974). As análises foram realizadas no Centro de Energia Nuclear na Agricultura (CENA), seguindo a metodologia descrita por Ducatti(1977).

Discussão dos resultados As amostras analisadas encontram-se descritas sucintamente na tabela 1 e são representadas graficamente na figura 3. Observa-se que as amostras de carbonatitos de Mato Preto se concentram fora do retângulo definido por Taylor et al. (1967), muito embora seja notável que a maior parte das mesmas se concentram num campo bem definido no qual se observa uma correlação positiva entre os valores de $\delta \mathrm{C}^{13}$ e $\delta \mathrm{O}^{18}$. Uma única amostra situa-se próxima ao retângulo de Taylor e representa provavelmente um carbonatito no qual as interações com fluidos tardios foram pouco importantes. Com relação às demais, que representam carbonatito intemperizado e fonólito carbonatizado, os valores isotópicos se apresentam com dispersão bastante acentuada. As duas amostras de mármores do Grupo Açungui foram analisadas para efeito de comparação com os carbonatitos e revelaram os mais elevados valores de $\delta \mathrm{O}^{18}$

A correlação positiva entre os valores de $\delta \mathrm{O}^{18}$ e $\delta \mathrm{C}^{13}$ no gráfico da figura 3 reflete um processo de diferenciação durante a evolução do magma carbonatítico. Verifica-se, no entanto, que as amostras de carbonatíto calcítico analisadas são representativas de uma única geração de carbonatitos cujas características principais são sua composição dominantemente calcítica e o fato de ocorrerem na matriz de brechas de conduto e na forma de diques. Assim, não há evidências de campo ou petrográficas que sustentem a existência de mais de um pulso de carbonatíto calcítico, levando a crer que o comportamento dos dados de isótopos estáveis é muito mais o reflexo de interações entre fluidos tardios e o magma carbonatítico do que propriamente de várias gerações de pulsos magmáticos.
Tabela l-Análises de $\delta O^{18}$ e $\delta \mathrm{C}^{13}$ dos carbonatitos do Complexo de Mato Preto e dos mármores do Grupo Açungui Table $1-\delta \mathrm{O}^{18}$ and $\delta \mathrm{C}^{\mathrm{B}}$ analyin for carbonatites of Mato Preto Complex and for marbles of Grupo Açungui

\begin{tabular}{|c|c|c|c|c|c|}
\hline \multirow[b]{2}{*}{ MMOSTRA } & \multicolumn{2}{|c|}{ CALCITA } & \multicolumn{2}{|c|}{ DOLOMITA } & \\
\hline & \begin{tabular}{|l|}
5018 \\
5 siow \\
\end{tabular} & $60 \$_{00}^{3}$ & 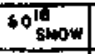 & ${ }_{0.600}$ & \\
\hline P TED & 11.22 & +2.40 & & & \\
\hline 540097 & 13.22 & -0.95 & & & \\
\hline 0740 & 15.21 & .0 .01 & & & \\
\hline 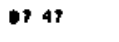 & 12,01 & .0 .10 & & & \\
\hline 4054 & 13,09 & -0.09 & & & \\
\hline 100846 & 15.55 & -0.14 & & & \\
\hline P\&6. & 11,44 & $-2,26$ & & & 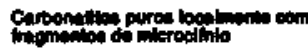 \\
\hline 7004 & 13.51 & $-0,06$ & & & \\
\hline pt2c & 1000 & -3.32 & & & \\
\hline TNA & 13.74 & +1.97 & & & \\
\hline THI & 13,69 & $-1,90$ & & & \\
\hline P37c & 13.21 & -1.90 & & & \\
\hline estec & 14.19 & -2.66 & & & \\
\hline Pusc & 14.01 & $-1,00$ & & & \\
\hline - 35. & 12,40 & $-2,6$ & & & \\
\hline p9sb & 13,94 & -942 & & & \\
\hline 67640 & 145 & .0 .40 & & & \\
\hline B76+6 & 14,15 & $-0,50$ & $11, \infty$ & $-2,70$ & 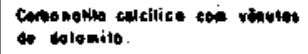 \\
\hline 87896 & $12, \infty 0$ & -2.50 & $m, \infty$ & $-2,+0$. & \\
\hline F 594 & 13.29 & 0.41 & & & \\
\hline F sso & 19,22 & $-0,73$ & & & \\
\hline $4+4$ & 23.12 & -6.52 & & & Carhanetito intumperizade. \\
\hline P654 & 11.6 & -2.16 & & & \\
\hline 194 & 15.15 & $-0.7 t$ & & & Hrescho ininnive. \\
\hline 6751 & \begin{tabular}{|l}
14,49 \\
12,30
\end{tabular} & $\begin{array}{l}-5.24 \\
-2.40\end{array}$ & 10,60 & -2.40 & Fonbling porlitititeo a merasta. \\
\hline 0754 & 11.90 & -2.70 & 13,40 & -1.00 & 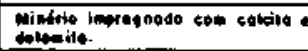 \\
\hline $\begin{array}{l}\text { ACungun I } \\
\text { Acuna ut } 2\end{array}$ & $\begin{array}{l}24,44 \\
22,60\end{array}$ & \begin{tabular}{|l|}
$-8,80$ \\
$-1,54$
\end{tabular} & & & 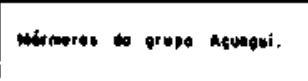 \\
\hline
\end{tabular}

Os dados de isótopos de $\mathrm{C}$ e $\mathrm{O}$ estão coerentes com o modelo de evolução de magmas carbonatíticos proposto por Pineau \& Javoy (1969) e Pineau et al. (1973), principalmente se forem consideradas somente as amostras coletadas na estrutura circular localizada no norte do complexo. A inclinação da reta ao longo da qual se distribuem os dados de $\mathrm{C}$ e $\mathrm{O}$ é, segundo Pineau et al. (1973), dependente da razão $\mathrm{H}_{2} \mathrm{O} / \mathrm{CO} 2$ da fase fluida e da temperatura de cristalização do magma. É notável ainda que algumas amostras se encontram levemente enriquecidas em $\delta \mathrm{O}^{18}$ sem apresentarem variação apreciável de $\delta \mathrm{C}^{13}$. Estas amostras, coletadas principalmente nas proximidades do depósito de fluorita Clugger e da falha de Morro Agudo, refletem provavelmente uma maior interação entre os carbonatitos e águas meteóricas circulantes ao longo da falha de Morro Agudo.

A distribuição dos valores de isótopos estáveis no diagrama da figura 3 tem sido correlacionado por alguns autores (Deines \& Gold 1973, Suwa et al. 1975) com a profundidade e condições de alojamento dos complexos carbonatíticos. Assim, Suwa et-al. 1975, baseando-se na classificação dos complexos carbonatíticos em associação vulcânica, sub vulcânica-vulcânica e subvulcânica, mostram que nas duas primeiras associações os valores de $\delta \mathrm{O}^{18}$ e $\delta \mathrm{C}^{13}$ apresentam uma variação 
acentuada devido a interações com o oxigênio atmosférico ou águas meteóricas, enquanto na terceira associação, os valores isotópicos exibem pequena dispersão. Se compararmos os dados de Mato Preto com os apresentados por estes autores, conclui-se que o Complexo de Mato Preto apresenta características iso tópicas de natureza vulcânica-sub vulcânica, ou mesmo vulcânica, haja vista o alto grau de dispersão dos isótopos de $\mathrm{C}$ e $\mathrm{O}$.

Faz-se necessário, também, comparar o Complexo de Mato Preto com outras estruturas alcalinas, particularmente com os carbonatitos da Fazenda Varela para os quais se encontram disponíveis dados de isótopos de C e O (Scheibe 1979). À semelhança de Mato Preto, as amostras de carbonatito fresco da Fazenda Varela dispõem-se segundo uma tendência com relação positiva entre os valores de $\delta \mathrm{C}^{13}$ e $\delta \mathrm{O}^{18}$ (Fig. 3), refletindo provavelmente a destilação de Rayleigh. Os carbonatitos de Lages exibem valores mais baixos de $\delta \mathrm{O}^{18}$ e $\delta \mathrm{C}^{13}$, cabendo ressaltar que seus pontos no diagrama da figura 3 encontramse no prolongamento da linha de evolução dos carbonatitos de Mato Preto.

Com relação ao carbonatito proterozóico de Angico dos Dias (Torquato 1987), localizado próximo à divisa dos Estados do Piauí e Bahia, observa-se que os valores isotópicos são significativamente diferentes dos dois carbonatitos mencionados acima. As poucas análises realizadas não exibem qualquer tendência de diferenciação e concentram-se num campo bem definido (Fig. 3).

Composição isotópica da calcita e dolomita coexistentes Foram selecionadas quatro amostras para análise da calcita e da dolomita coexistentes pelo método da extração dupla de Epstein et al. (1964). Os dados obtidos encontram-se representados graficamente na figura 4 e mostram que, na maior parte das amostras, os valores de $\delta \mathrm{O}^{18}$ e $\delta \mathrm{C}^{13}$ das calcitas são maiores que os nas dolomitas coexistentes. Segundo Garlick (1969 in Hoefs 1987), a dolomita, quandj|ém equilíbrio isotópico com a calcita, apresenta valores de $\delta \mathrm{O}^{18}$ mais elevados, refletindo características cristaloquímicas inerentes aos dois carbonates. $\mathrm{O}$ enriquecimento em $\delta \mathrm{O}^{18}$ na dolomita, comparativamente à calcita, é, segundo Deines (1970, p. 1221), um fenônemo comum em carbonatitos. Quando em equilíbrio isotópico, estes dois minerais apresentam uma diferença em $\delta \mathrm{C}^{13}$ de aproximadamente $1 \%$ o a $2 \%$ o.

Os dados obtidos em Mato Preto mostram então que os dois carbonates analisados encontram-se em desequilíbrio isotópico, o que reforça as conclusões de Santos (1988) e Santos \& Dardenne (1988), que descrevem, com base em critérios petrográficos e texturais, a existência de duas gerações carbonatito na área do depósito de fluorita Clugger. A geração mais antiga possui composição dolomítica e constitui parte dos fragmentos das brechas, enquanto a geração mais jovem possui composição dominantemente calcítica e ocorre na forma de diques ou na matriz de brechas de conduto.

Variação dos isótopos estáveis de C e O com a profundida$d e$ Para avaliar as variações dos valores de $\delta \mathrm{C}^{13}$ e $\delta \mathrm{O}^{18}$ com a profundidade, foram utilizadas amostras do testemunho de sondagem DB 47, localizado na porção S W do depósito Clugger. Foram analisadas nove amostras cujas posições no furo de sondagem, bem como uma descrição sucinta dos litotipos, encontram-se na figura 5. Na figura 6, estão representados graficamente a profundidade de coleta das amostras versus os valores de $\delta \mathrm{C}^{13}$ e $\delta \mathrm{O}^{18}$ dos carbonatitos. Observando-se este diagrama verifica-se que, à exceção de duas amostras que representam um carbonatito intemperizado e um fonólito carbonatizado, os dados isotópicos apresentam correlação positiva e permanecem mais ou menos constantes até cerca de $135 \mathrm{~m}$ de profundidade.

Em duas amostras alteradas, o comportamento dos isótopos de $\mathrm{C}$ e $\mathrm{O}$ é o inverso do que foi observado nas rochas

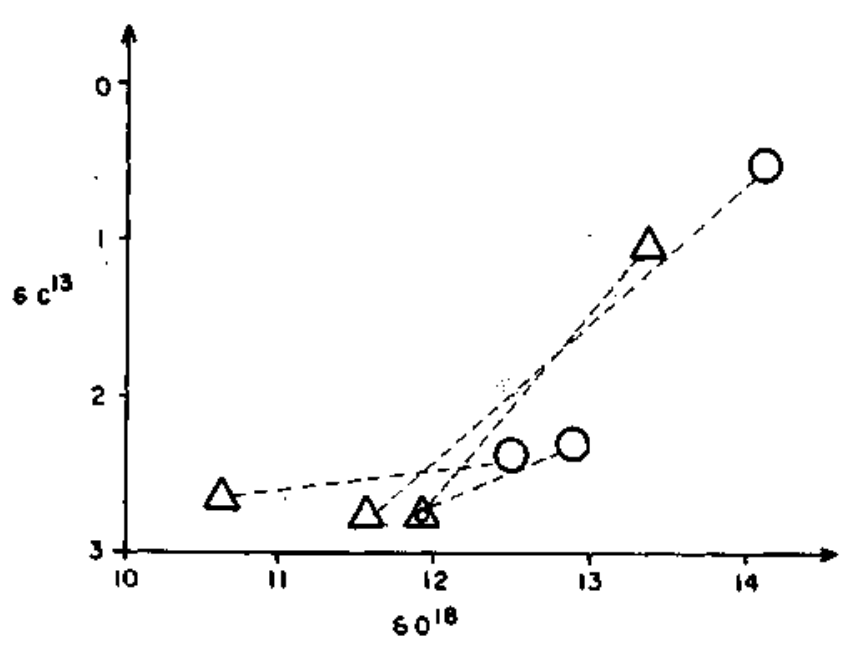

Figura 4 - Diagrama $\delta O^{18}$ versus $\delta \mathrm{C}^{13}$ para dolomita e calcita coexistentes, analisadas pelo método da extração dupla de Epstein et al. (1964). Linhas tracejadas = pares calcita-dolomita analisadas; $\Delta=$ dolomita; $\mathrm{O}=$ calcita" Figure $4-\delta \mathrm{O}^{18} \times \delta \mathrm{C}^{13}$ diagram for coexistent dolomite and calcite, analysed by Epstem et al. (1964) double extraction method. In the diagram lives $=$ calcite-dolomite; $\Delta=$ dolomite, and $\mathrm{O}=$ calcite

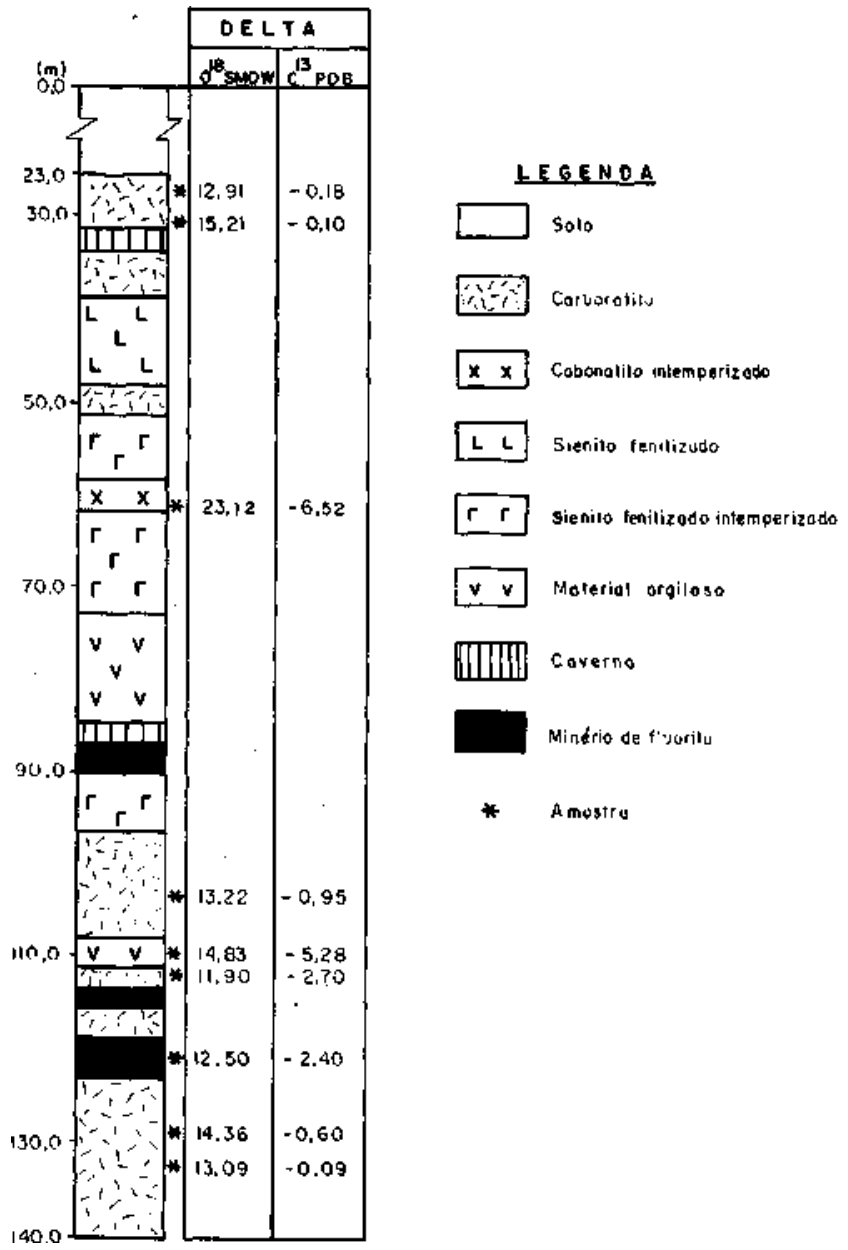

Figura 5 - Descrição sucinta da sondagem DB47 com o posicionamento das amostras analisadas para isótopos de $\mathrm{Ce} O$ Figure 5 - DB47 $\log$ and location of samples analysed for $\mathrm{C}$ and $\mathrm{O}$ isotopes 
frescas. Um fenômeno semelhante também foi observado por Hubberten et al. (1988) nos diques de alkivito do Complexo Carbonatítico de Kaisertuhl, Alemanha. Neste caso, entretanto, os autores consideram que a cristalização dos carbonates deu-se num sistema fechado e que o comportamento dos isótopos de $\mathrm{C}$ e $\mathrm{O}$ pode ser explicado por meio do sistema $\mathrm{CaCO}_{3}-\mathrm{CO}_{2}-\mathrm{H}_{2} \mathrm{O}$. Segundo os autores, que utilizam os dados de Bottinga (1969), no sistema fechado $\mathrm{CaCO}_{3}-\mathrm{CO}_{2}$, o isótopo de carbono mais pesado particiona para a fase fluida $\left(\mathrm{CO}_{2}\right)$, enquanto que no sistema $\mathrm{CaCO}_{3}-\mathrm{H}_{2} \mathrm{O}$ o isótopo de oxigênio mais pesado particiona para o carbonato, estabelecendo-se a partir daí um comportamento inverso entre os dois elementos. Esta explicação seria viável para explicar os isótopos de $\mathrm{C}$ e $\mathrm{O}$ na amostra de fonólito carbonatizado, mas seria bastante improvável para explicar a amostra de carbonatito intemperizado. É de se esperar que neste último caso a troca isotópica tenha ocorrido num sistema aberto e com abundância de água meteórica, o que está coerente com o aumento dos valores de $\delta \mathrm{O}^{18}$. Uma possível explicação para o comportamento dos isótopos de $\mathrm{C}$ estaria nos processos de dissolução e reprecipitação dos carbonates.

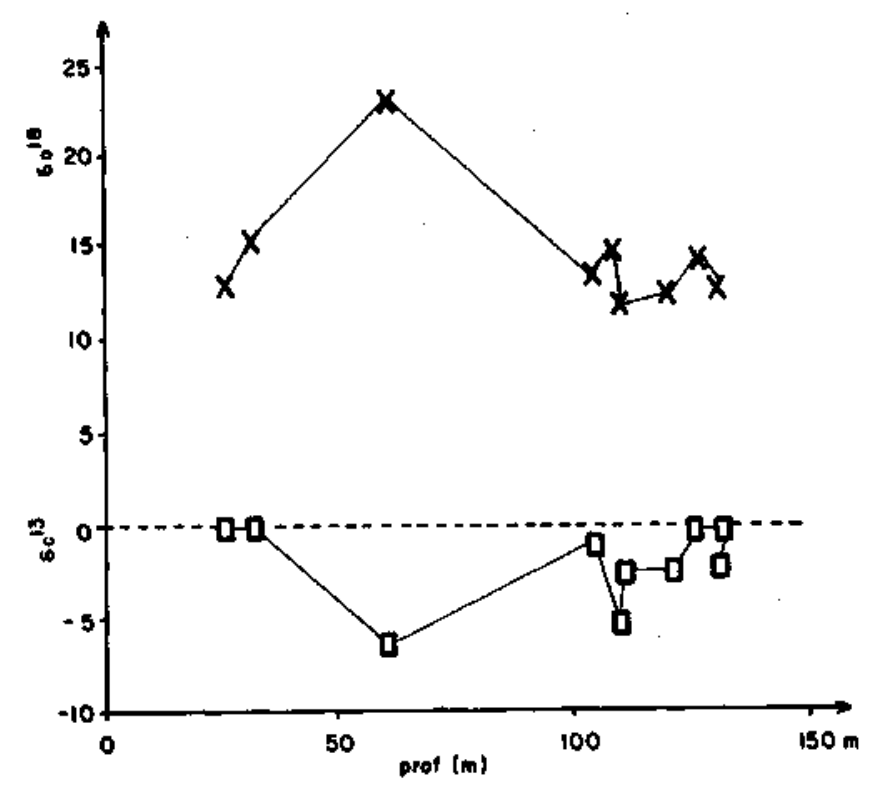

Figura 6 - Diagrama da profundidade (m) versus valores de $C$
$e O$ obtidos a partir de amostras de testemunho de sondagem
DB47. Simbologia: $X=8 O^{18} ; \square=8 C^{13}$
Figure 6 - Plot of $C$ and $O$ values taken from samples of $\mathrm{DB} 47$.
Symbols: $\mathrm{X}=6 \mathrm{O}^{18} ; \square=6 \mathrm{C}^{13}$

Distribuição especial dos valores isotópicos A figura 3 permite observar, também, as variações isotópicas dos carbonatitos do Mato Preto em três áreas distintas, orientadas grosseiramente segundo a direção NW, ou seja, paralelamente ao eixo de distribuição das estruturas circulares e perpendicularmente à falha de Morro Agudo. Ao se observar a figura, podem ser levantadas as seguintes indagações: 1. quais são as diferenças isotópicas entre os carbonatitos próximos ao contato do Granito Três Córregos e aqueles localizados nas proximidades dos metassedimentos do Grupo Açungui? e 2. qual o comportamento de $\delta \mathrm{C}^{13}$ e $\delta \mathrm{O}^{18}$ na área mineralizada, e particularmente nos proximidades da falha de Morro Agudo?
Comparando-se o grupo de amostras coletadas no extremo $\mathrm{N}$ W do complexo, ou seja, nas proximidades do contato com o Granito Três Córregos com o do extremo SE, próximo ao contato com os metassedimentos Açungui, observam-se diferenças pouco significativas, principalmente nos valores de $\mathrm{C}$. As amostras do primeiro grupo apresentam valores de $\delta \mathrm{O}^{18}$ de $+12 \%$ o, o que de certa forma é compatível com a proximidade das rochas graníticas. Segundo Taylor (1968), os valores de $8 \mathrm{O}^{18}$ para granitos situam-se próximos a $+10 \%$. Quanto às amostras do segundo grupo, os valores de $8 \mathrm{O}^{18} \mathrm{si}$ tuam-se em geral acima de $+13,5 \%$ o. Se se levar em conta que em duas amostras de mármores do Grupo Açungui foram obtidos valores iguais a $+22,6 \% \mathrm{o}$ e $+24,9 \% \mathrm{o}$, sugere-se a possibilidade de uma troca isotópica entre os carbonatitos e as rochas desta unidade.

Os valores de $\mathrm{SC}^{13}$ são mais difíceis de serem interpretados, tendo sido observado que em geral são maiores para as amostras da porção SE do complexo. Mesmo uma comparação com os mármores do Grupo Açungui torna-se difícil, uma vez que as poucas amostras analisadas desta unidade exibem valores de $-8 \%{ }_{0} \mathrm{e}+2 \% \%_{0}$.

Com relação às amostras coletadas na área do depósito de fluorita Clugger e nas proximidades da falha de Morro Agudo, observa-se que, apesar de estarem de acordo com a tendência de destilação de Rayleigh, verifica-se uma maior dispersão nos valores de $\delta \mathrm{O}^{18}$ se comparados a outras áreas do complexo. Isto reflete provavelmente a maior interação com águas ao longo da falha de Morro Agudo. Chai (1974, In: Criss et al. 1984) mostra que o equilíbrio isotópico entre a calcita e um fluido depende em grande parte do grau de "trituração" ou "pulverização" da rocha. Nesse sentido, tanto as condições de "trituração" quanto de percolação de fluidos rneteóricos podem ser satisfeitas ao longo da falha de Morro Agudo.

CONCLUSÕES Os dados isotópicos de carbono e oxigênio de carbonates dos carbonatitos de Mato Preto são significativamente diferentes dos metassedimentos do Grupo Açungui.

Com relação aos dados de isótopos estáveis apresentados pela literatura, o Complexo de Mato Preto pode ser considerado de natureza vulcânica-subvulcânica. Observa-se um nítido processo de diferenciação isotópica que obedece à destilação de Rayleigh, proposta por Pineau \& Javoy (1969) para a evolução isotópica de magmas carbonatíticos.

Verificam-se, ainda, variações inexpressivas dos valores de $\delta \mathrm{O}^{18}$ e $\delta \mathrm{C}^{13} \mathrm{com}$ a profundidade e que as amostras que exibem alterações hidrotermais ou intempéricas tendem a apresentar uma correlação positiva entre os isótopos de $\mathrm{C}$ e $\mathrm{O}$.

Os carbonatitos dolomíticos, que constituem um dos pulsos magmáticos, mostram valores de isótopos de $\mathrm{C}$ e $\mathrm{O}$ mais pesados que as calcitas coexistentes, deixando clara a existência de um desequilíbrio isotópico entre ambos. A maior parte da calcita é formada durante o segundo pulso carbonatítico, que é dominantemente calcítico.

Observa-se também que, nas proximidades da falha de Morro Agudo e junto às concentrações de fluorita, os valores de $\mathrm{SO}^{18}$ tendem a ser mais dispersos que em outras áreas do complexo, sugerindo uma maior interação com fluidos meteóricos.

Agradecimentos Os autores agradecem à Du Pont do Brasil, em especial a Moacir Almeida e Robert Jenkins, e ao laboratório de isótopos estáveis do CENA/USP, em Piracicaba, em especial à técnica Maria Antônia Zambetta Perez. Agradecem também aos professores Reinhardt Adolfo Fuck e José Carlos Gaspar, da UnB, pelas sugestões e discussões sobre o texto. Este trabalho é parte da Dissertação de Mestrado de um dos autores, que teve o apoio do Conselho Nacional de Desenvolvimento Científico e Tecnológico - $\mathrm{CNPq}$ (proc. 40.0726/86) e do PADCT. 


\section{REFERÊNCIAS BIBLIOGRÁFICAS}

ALMEIDA, F.F.M. 1983. Relações tectônicas das rochas alcalinas mesozóicas da região meridional da plataforma sul-americana. Rev. Bras. Geoc., 13(3):139-158.

BOTTINGA, Y. 1969. Calculated fractionation factors for carbon and hydrogen isotope exchange in the system calcite-carbon dioxide-graphite-methane-hydrogen-water vapor. Geoch. Cosmoch.Acta, 33:49-64.

CORDANI, U.G. \& HASUI, Y. 1968. Idades de K-Ar de rochas alcalinas do primeiro planalto do Estado do Paraná. In: CONG. BRAS. GEOL., 29, Ouro Preto, 1976. Anais... Ouro Preto, SBG. v. 1 , p. $139-153$.

CRISS, R.E.; GREGORY, R,T.; TAYLOR, H.P. 1984. Kinetic theory of oxygen isotopic exchange between minerals and water. Geoch. Cosmoch. Acta, 51(5):1099-1108.

DEINES, P. 1970. The carbon and oxygen isotopic composition of carbonates from Oka Carbonatite Complex., Quebec, Geoch. Cosmoch.Acta, 34:1199-1225.

DEINES, P. \& GOLD, D.P. 1973. The isotopic composition of carbonatite and kimberlite carbonates and their bearing on isotopic composition of deep-seated carbon. Geoch. Cosmoch. Acta composition $37: 1707-1733$.

DENAYER, M.E. 1970. Rapports isotopiques O e C et condition d'affleurement dês carbonatites de 1'Affrique Central. Compt. Rend., 270D:2155-2158.

DUCATTI, C. 1977. Medidas da variação natural isotópica $\mathrm{C}^{13} / \mathrm{C}^{12} \mathrm{em}$ amostras orgânicas. Piracicaba. 118p. (Dissertação de Mestrado, CENA-USP)

EPSTEIN, S; GRAF, D.L.; DEGEN, E.T. 1964. Oxygen isotope studies on the origen of dolomites. In: H. CRAIG; S.L. MILER \& G.J. WASSERBURG, eds. Isotopic and Cosmic Chemistry. p. $169-180$

FIORI, A.P. 1985. As falhas da Lancinha e de Morro Agudo e estruturas secundárias associadas. In: SIMP. SUL-BRAS. GEOL., 2. Florianópolis, 19S5.Atas... Florianópolis, SBG. p. 146-158.

HOEFS, J. 1987. Stable isotope geochemistry. Berlin, Springer Verlag. $241 \mathrm{p}$.

HUBBERTEN, H.W.; KATZ-LEHNERT, K.; KELLER, J. 1988. Carbon and oxygen isotope investigation in carbonatites and related rocks from the Kaisertuhl, Germany. Chem. GeoL, 70:257-274.

HUTCHISON, C.S. 1974. Laboratory handbook of petrografic tecnhiques. New York, Wiley \& Sons. 527 p.

JENKINS, R.E. 1987. Geology of the Clugger-Fluorite Deposit, Mato Preto, Paraná, Brazil. Rev. Bras. Geoc. 17(3):288-294.

LOUREIRO, F.E.L. \& TAVARES, J.R. 1983. Duas novas ocorrências de carbonatitos: Mato Preto e Barra do Rio Itapirapuã. Rev. Bras. Geoc., 13(1):7-11.

NIELSEN, T.F.D. \& BUCHARDT, D. 1975. Sr-C-O isotopes in nephelinitic rocks and carbonatites, Gardiner Complex, Tertiary of East Greenland. Chem. Geol., 53:207-217.
PILLOT, J. 1974. Les isotopes en geologic (methodes et applications). Paris, Doin S/A. $176 \mathrm{p}$.

PINEAU, F. \& JAVOY, M. 1969. Determination des rapports isotopique $0^{18} / 0^{16}$ e $\mathrm{C}^{13} / \mathrm{C}^{12}$ dans diverges carbonatites. Implications genetiques. Compt. Rend., 269D: 1930-1933.

PINEAU, F.; JAVOY, M.; ALLEGRE, C.J. 1973. Etude systematique des isotopes de 1'oxygene, du carbone e du strontium dans les carbonatites. Geoch. Cosmoch. Acta, 37:2363-2377.

SANTOS, R.V. 1988. Geologia e geoqufmica do depósito de fluorita do Complexo AlcaUno Carbonatitíco de Mato Preto, Paraná. Brasília. 144p. (Dissertação de Mestrado, UnB, inédita).

SANTOS, R.V.\& DARDENNE, M.A. 1988. Fluorita de Mato Preto: um caso particular de mineralização de fluorita associada complexo alcalino carbonatftico. In: CONG. BRAS. GEOL., 35, Belém, 1988. Anais... Belém, SBG. v. 3, p. 1251-1261.

SCHEIBE, L.F. 1979. Estudo petrológico e geoqu\&nico dos carbonatitos da Fazenda Varela, Lages, Santa Catarina, Brasil. Porto Alegre. 135 p. (Dissertacão de Mestrado, UFRGS). (Inédita).

SUWA, K; OANA, J.S.; WADA, H ; OSAKI, S. 1975. Isotope geochemistry and petrology of African carbonatites. Pergamon Press. Physics and Chemistry of the Earth, 9:735-745.

TAYLOR, H.P. 1968. The oxygen isotopic geochemistry of igneous rocks. Contr. Min. Petrol., 19:1 -71.

TAYLOR, H.P.; FRECHEN, J; DEGENS, E.T. 1967. Oxygen and carbon isotope studies of carbonatite from the Leacher See district, West Germany and the Alno district, Sweeden. Geoch. Cosmoch. Acta, 31:407-430.

TORQUATO, J.R. 1987. Geoqufmica isotópica de carbonatitos. 59 p. (Inédito).

TREIN, E.; MARINI, O.J.; \& FUCK, R.A. 1966. Rochas alcalinas do primeiro planalto do Estado do Paraná. In: BIGARELA, SALAMUNI \& PINTO, ed., Geologia do Pré-Devoniano e intrusivas subjacentes da porção oriental do Estado do Paraná. Curitiba, p. 325-347. (Boi. Paranaense de Geoc., 23/25).

ULBRICH, H.G J. 1986. As brechas de origem fgnea: revisão e proposta para uma classificação. São Paulo, IG/USP. p. 1-82. (Boi., Publ. Esp.,3)

WHITE, D.E. 1974. Diverse origins of hydrotermal ore fluids. Econ. Geol., 69:954-973.
MANUSCRITO A602

Recebido em 23 de maio de 1989

Revisão do autor em 22 de agosto de 1990 Revisão aceita em 27 de agosto de 1990 